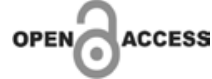

\title{
Linfadenectomia Pélvica de Resgate Radioguiada - Uma Nova Ferramenta para Detecção e Tratamento de Metástases no Câncer de Próstata Recorrente
}

\author{
Radioguided Rescue Pelvic Lymphadenectomy - A New Tool for \\ Detection and Treatment of Metastases in Recurrent Prostate \\ Cancer
}

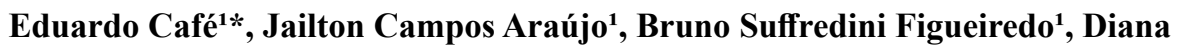 \\ Solano de Freitas Souza ${ }^{2}$, Adelina Sanches ${ }^{2}$ \\ ${ }^{1}$ Serviço de Urologia; ${ }^{2}$ Serviço de Medicina Nuclear; Hospital Santa Izabel, Santa Casa de \\ Misericórdia da Bahia; Salvador, Bahia, Brazil
}

Correspondence addresses:

Dr. Eduardo Café

eduardocafe@gmail.com

Received: April 9, 2021

Revised: May 20, 2021

Accepted: June 15, 2021

Published: June 30, 2021

Data Availability Statement: All relevant data are within the paper and its Supporting Information files.

Funding: This work was the result of authors' initiative. There was no support of research or publication funds.

Competing interests: The authors have declared that no competing interests exist.

Copyright

(C) 2021 by Santa Casa de Misericórdia da Bahia.

All rights reserved.

ISSN: 2526-5563

DOI: 10.35753
O câncer de próstata é o tumor mais comum no homem, e mesmo após a terapia inicial otimizada, uma parte importante da população pode apresentar recidiva do tumor. A identificação de sítios de metástases por meio da medicina nuclear é algo já solidificado e de alta eficiência, sendo que o uso dessa tecnologia com um probe pode extrapolar sua utilização, antes apenas diagnóstica, para ainda atuar como modelo experimental na área terapêutica. Método. Relato de caso de paciente de 79 anos com diagnóstico inicial de câncer de próstata, PSA $9.4 \mathrm{ng} / \mathrm{mL}$, achado em biópsia como Gleason $7(3+4)$. Foi submetido à prostatectomia radical com linfadenectomia em 2010. Evoluiu sem intercorrências até que em 2014 foi diagnosticada recidiva bioquímica, sendo então submetido à radioterapia de resgate. Após 2 anos de radioterapia, voltou a apresentar nova elevação do PSA e ao PET-PSMA evidenciou-se aumento dos linfonodos pélvicos. Foi submetido a protocolo experimental de linfadenectomia pélvica radioguiada com PSMA - 99mTc. Embora seja uma tecnologia recente, a cirurgia radioguiada é uma forma eficaz de identificar pequenas metástases em pacientes submetidos à linfadenectomia de resgate, auxiliando o cirurgião na detecção de linfonodos doentes, inclusive alguns que passariam desapercebidos sem o probe.

Palavras-chave: Câncer de Próstata; Cirurgia Radioguiada; Linfadenectomia Pélvica.

Prostate cancer is the most common tumor in man. After initial therapy, part of the affected population also presents recurrence. The identification of metastasis sites through this nuclear medicine exam allows the extrapolation of its use, previously only diagnostic, to still act as an experimental model in the therapeutic field case report of a 79 years old patient with an initial serum PSA of $9.4 \mathrm{ng} / \mathrm{mL}$ was diagnosed with prostate cancer with a biopsy showing a Gleason $7(3+4)$. He underwent radical prostatectomy with standard lymphadenectomy in 2010 . It evolves uneventfully until 2014 a biochemical recurrence was diagnosed and was then submitted to salvage radiotherapy. After two years of radiotherapy, he returned to have a new elevation of PSA and a PET-PSMA, which showed pelvic lymph node enlargement. He was submitted in an experimental protocol of 
radioguided pelvic lymphadenectomy with PSMA - 99mTc. Although it is a recent technology, radioguided surgery is an effective way to identify small metastases in patients undergoing salvage lymphadenectomy, helping the surgeon to detect diseased lymph nodes and not adding morbidity and mortality to the procedure.

Keywords: Prostate Cancer; Radioguided Surgery; Pelvic Lymphadenectomy.

\section{Instrodução}

O câncer de próstata é o tumor mais frequente no homem, e mesmo após as terapias iniciais uma boaparte dapopulaçãomasculina acometidaainda apresenta recidiva deste tumor. ${ }^{1,2}$ No contexto de recorrência local do tumor, o tratamento pode envolver a radioterapia ou cirurgia de resgate. Os pacientes com disseminação metastática geralmente recebem terapia de privação de androgênica. ${ }^{3}$ Naqueles pacientes com baixa carga tumoral e que apresentem um bom estado de saúde, podem se beneficiar de tratamentos de salvamento local, influenciando positivamente a sobrevida livre de progressão e postergando o início de terapias sistêmicas mais agressivas. ${ }^{4,5}$

Os gânglios linfáticos representam um dos locais mais frequentemente afetados pelo câncer de próstata recorrente. Originalmente, a arquitetura linfática é preservada na vigência do câncer de próstata, mas ela pode variar significativamente entre indivíduos e sofrer alterações ou desvios secundário a bloqueios tumorais em sua vianormal.Após a prostatectomia radical ou radioterapia, alterações importantes podem ocorrer nas vias de drenagem linfática. Por esse motivo, as metástases podem surgir em locais inesperados e fora do campo clássico de dissecção linfonodal pélvica. Reconhecer o estado exato e a extensão da disseminação linfática representa um desafio complexo para os médicos. ${ }^{6,7}$

Nesse contexto, o campo da imagem de tomografia com emissão de pósitrons (PET) avançou substancialmente com o uso de marcadores baseados em colina e, especialmente, marcadores direcionados contra o antígeno de membrana específico da próstata (PSMA). A identificação dos sítios de metástase através desse exame permite a extrapolação do seu uso, anteriormente apenas diagnóstico, para atuar ainda como modelo experimental, no campo terapêutico. O uso do gama probe para identificação dos marcadores de membrana (PSMA) no intra-operatório permite a localização mais acurada dos linfonodos acometidos.

\section{Relato de Caso}

Paciente masculino, 79 anos, com diagnóstico em 2010 de câncer de próstata Gleason $7(3+4)$, com PSA inicial de 9,4 ng/mL, foi submetido à prostatectomia radical com linfadenectomia padrão ainda em 2010. O resultado da anatomia patológica dapeça cirúrgicaevidenciou: Gleason 7 $(4+3)$, margens livres, $\mathrm{T} 2 \mathrm{cN} 0 \mathrm{M} 0$, sem linfonodos acometidos. Evoluiu sem intercorrências até que em 2014 foi diagnosticada recidiva bioquímica, sendo então submetido à radioterapia de resgate (7000GY, 35 seções), com nadir em 0,25 em maio de 2015. Após 2 anos de realizada a radioterapia retornou a ter nova elevação de PSA, sendo que em 2019 teve duplicação menor que 6 meses. Foi re-estadiado com auxílio do PET/CT-PSMA, o qual demonstrou linfonodomegalia pélvica na cadeia ilíaca externa direita, medindo $2,5 \mathrm{~cm}$ nos maiores eixos, coexistindo outro linfonodo de $0,5 \mathrm{~cm}$ imediatamente abaixo da bifurcação da artéria ilíaca comum homolateral, sem outras lesões à distância. Como o paciente apresentava bom status clínico e sem evidências de outros sítios de metástase, foi optado por inclusão no protocolo de cirurgia radioguiada com PSMA 99mTc.

\section{Descrição do Procedimento e Resultado}

Um kit HYNIC- PSMA - SN (NúcleoEquipo HYNIC- PSMA - SN) "nome Comercial" fabricado pelo ININ (Instituto Nacional de Investigaciones Nucleares) foi marcado com eluato de $99 \mathrm{mTc}$-Tecnécio, com 
controle de qualidade que garantia sua pureza radionuclídica. Foram injetados $740 \mathrm{MBq}$ do material e realizadas imagens após 2 e 22 horas, para garantir a adequada marcação com 99mTc-Tecnécio, dos linfonodos previamente vistos no PET/CT PSMA-Ga-68 (Figura 1). A cirurgia foi realizada pela via laparoscópica transperitoneal. Para tal procedimento, o paciente foi posicionado em decúbito dorsal horizontal com mesa em posição de Trendelemburg e utilizados cinco trocartes, incluindo um trocarte de $12 \mathrm{~mm}$ para o uso intraoperatório do gama-probe longo (Figura 2). Foi realizada a linfadenectomia pélvica direcionada para a região previamente identificada pelo PET/PSMA - região de ilíaca externa direita e bifurcação da ilíaca comum direita - e com o auxílio do gama-probe, identificamos os dois linfonodos acometidos. Foi realizada a exérese do tecido linfonodal. Amostras de tecido gorduroso sadio também foram retiradas para que ex vivo fosse feita também a comparação na obtenção dos sinais do gama-probe e para que, mais uma vez, confirmasse a retirada correta do tecido acometido (Figura 3). O valor do sinal emitido no tecido gorduroso serve como parâmetro de análise. É considerado como tecido doente, aquele que emite pelo menos o dobro do valor do tecido não acometido.

$\mathrm{O}$ paciente evoluiu sem intercorrências e recebeu alta menos de 24 horas após a cirurgia. $\mathrm{O}$ resultado do exame anatomo-patológico confirmou metástase de adencocarcinoma de próstata em ambos linfonodos. O exame de PSA de controle realizado após 45 dias da cirurgia foi de $0,87 \mathrm{ng} / \mathrm{mL}$.

Figura 1. Imagens da cintilografia com PSMA-99mTc de corpo inteiro em projeções anterior e posterior, demonstrando captação em linfonodo pélvico.

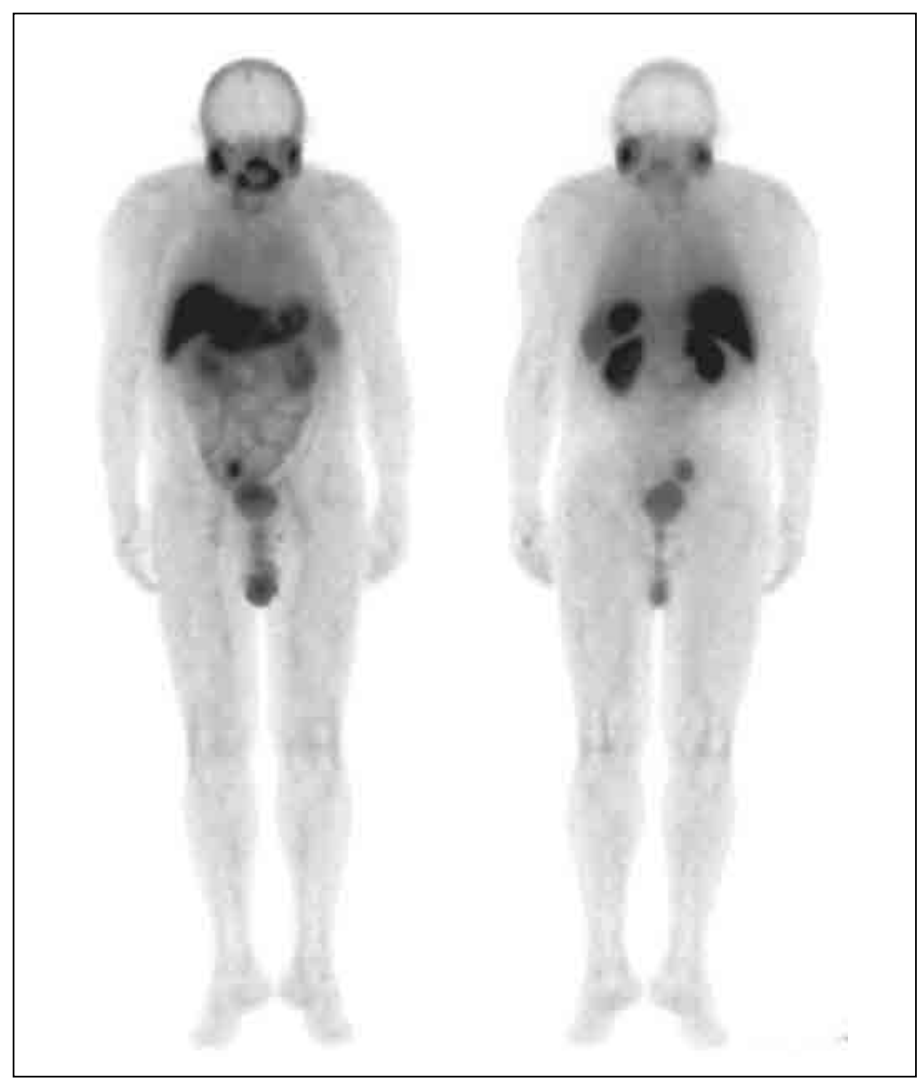


Figura 2. Posicionamento dos trocartes. Dois trocartes de $10 \mathrm{~mm}$ (ótica e pinça bipolar). Um trocarte de $12 \mathrm{~mm}$ para retirada das peças e passagem de probe. Dois trocartes de $5 \mathrm{~mm}$ para passagem de pinças acessórias.

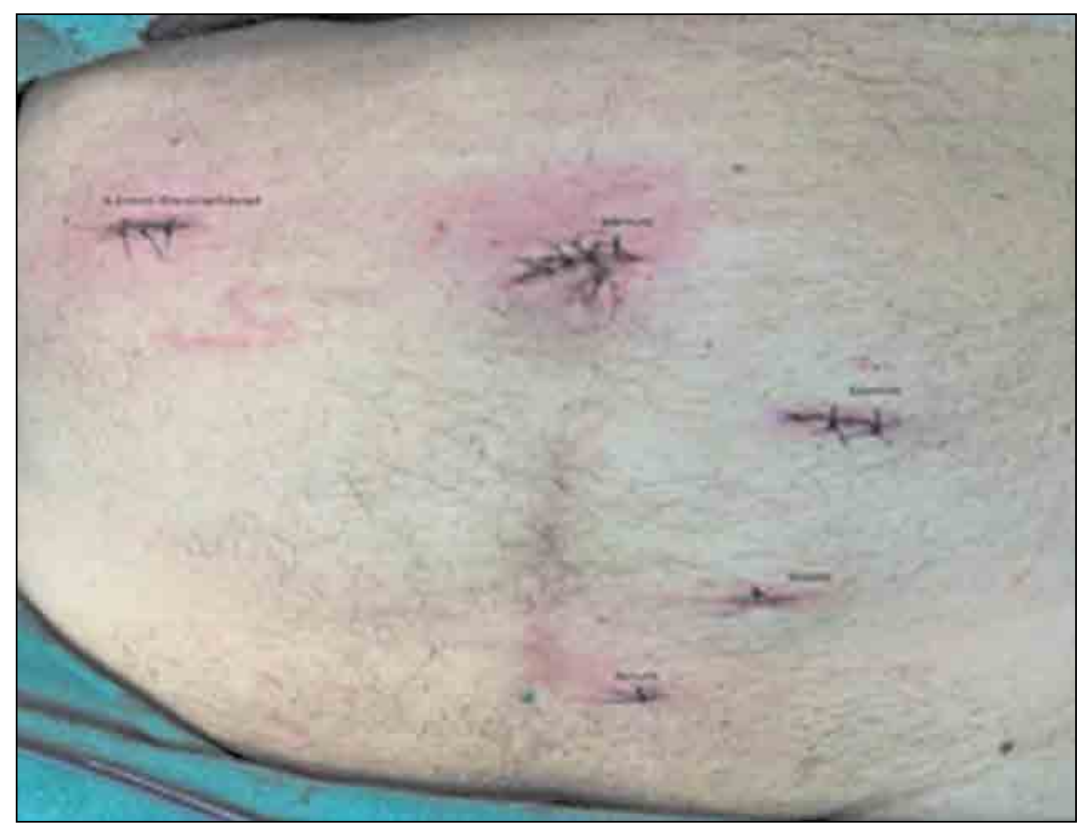

Figura 3. Probe mostrando 168 SUV em peça de produto de linfadenectomia pélvica.

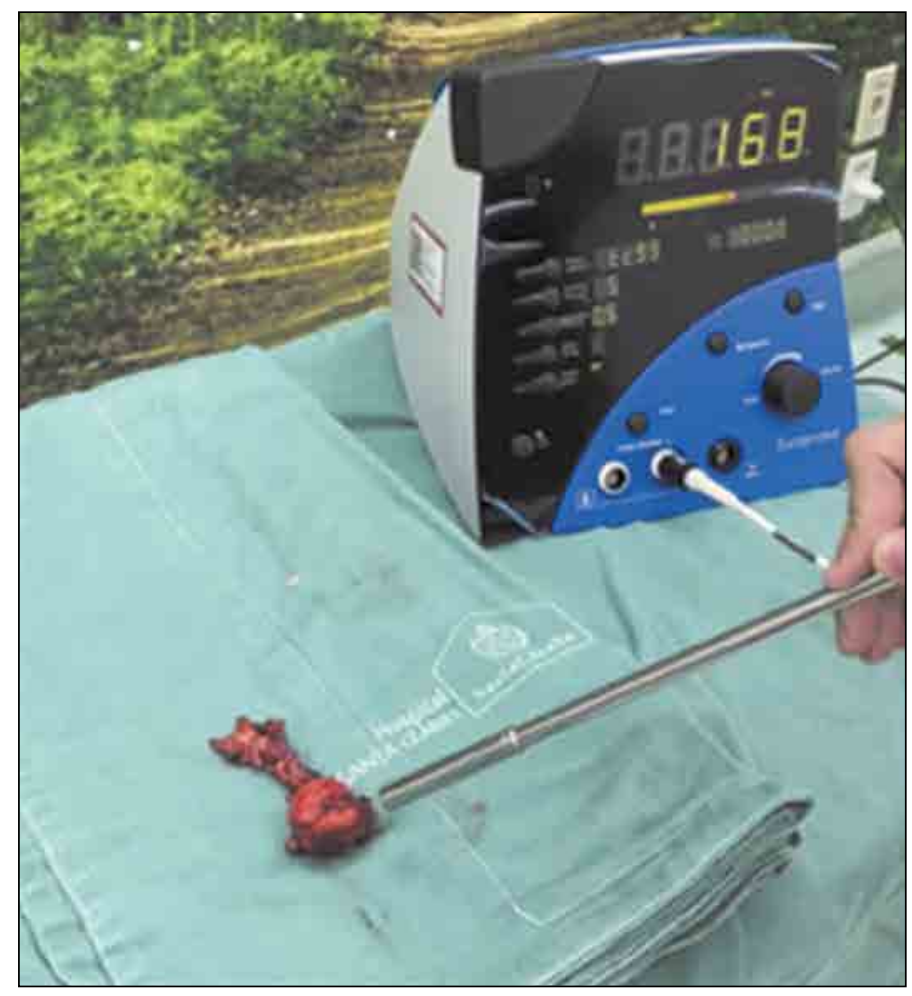




\section{Discussão}

Com o crescente uso do PET/PSMA em recidiva de câncer de próstata, os tratamentos direcionados a metástase, como a linfadenectomia de resgate, vem se tornando mais factíveis. No entanto, a identificação precisa de lesões pequenas e /ou atipicamente localizadas durante a linfadenectomia de resgate pode ser um desafio, considerando os tratamentos e manipulações previas. Nesse contexto, a cirurgia radioguiada pode ser uma vantagem. ${ }^{8}$ Esta cirurgia detecta recorrências de câncer de próstata expressando o PSMA com uma alta sensibilidade $(92,3 \%)$ e especificidade (93,5\%).

Maurer e colaboradores $(2018)^{9}$ foram capazes de mostrar em 31 pacientes que se submeteram à cirurgia radioguiada com 99mTC-PSMA que todas as lesões vistas anteriormente no exame de imagem com 68GaPSMA PET foram também identificadas no intra-operatório. Sendo que, em dois pacientes, a cirurgia radioguiada conseguiu identificar recorrências linfonodais de $3 \mathrm{~mm}$ não vistas no exame de imagem prévio. Rasucher e colaboradores (2017) também encontraram 5 lesões suspeitas não visualizadas no $\mathrm{PET} / \mathrm{CT}$ em $3 / 31$ pacientes. ${ }^{10}$

Ainda faltam dados de longo prazo para determinar se a terapia radioguiada de fato teria um impacto positivo tão maior quanto as terapias já em voga. Autilização do gama probe vem sendo investigada como ferramenta para auxiliar na retirada dos linfonodos acometidos. Os estudos já publicados mostram um valor preditivo positivo que varia de $88 \%$ a aproximadamente $100 \%$ ex vivo. ${ }^{9,10} \mathrm{O}$ estudo de Kniiper e colaboradores (2018), comparando pacientes submetidos à linfadenectomia convencional e radioguiada, encontraram, em termos de desfecho de curto prazo, um declínio de PSA em geral, declínio PSA $>50 \%$ e $>90 \%$ no prazo de 6 semanas sem tratamento adicional em 50\%, 29\% e 7\% no grupo convencional em comparação com $100 \%, 92 \%$ e $53 \%$ no grupo submetido à cirurgia radioguiada, respectivamente. ${ }^{8}$
Em consonância com a literatura, é fundamental a escolha cuidadosa no perfil de pacientes que serão submetidos a este procedimento a fim de que realmente traga benefício no controle oncológico e diminua a morbidade do tratamento. Um dos critérios de elegibilidade seria o PET/PSMA positivo, demonstrando doença loco-regional e ausência de metástases à distância. Outros fatores e dentre eles o status clínico do paciente e comorbidades associadas também devem ser levadas em consideração.

Rigatti e colaboradores ${ }^{11}$ observaram que a sobrevida sem recorrência clínica de 5 anos foi menor para pacientes com linfonodos positivos no retroperitôneo em comparação com os pacientes nos quais os linfonodos positivos foram detectados apenas na pelve $(11 \%$ vs $53 \%$; $\mathrm{P}<0.001$ ), assim como o nível de PSA $<4$ ng/ $\mathrm{mL}$ em comparação com PSA $\geq 4 \mathrm{ng} / \mathrm{mL}(48 \%$ vs 13\%). Pacientes com fatores desfavoráveis podem ter uma maior necessidade de tratamentos posteriores para controle da progressão clínica da doença.

Vários fatores pós-operatórios, incluindo resposta bioquímica completa e número de gânglios linfáticos positivos na linfadenectomia de salvamento, também são estabelecidos como preditores independentes de progressão clínica. ${ }^{10}$

$\mathrm{Na}$ revisão da literatura, não foram observadas complicações relacionadas à infusão do radiofármaco e complicações relacionadas ao procedimento cirúrgico na sua grande maioria foram classificadas na escala de ClavienDindo como grau 1 - linforréia (21\%), febre (25-30\%), íleo prolongado., ${ }^{410,11}$ Isso reforça a maior segurança e diminuição de complicações do procedimento em comparação com as linfadenectomias padrão.

\section{Conclusão}

A recidiva do câncer de próstata é um desafio para urologistas e oncologistas. Embora seja uma tecnologia recente, a cirurgia radioguiada é uma 
forma eficaz de identificar pequenas metástases em pacientes submetidos à linfadenectomia de resgate, auxiliando o cirurgião na detecção de linfonodos doentes e não agregando morbimortalidade ao procedimento. No curto prazo, parece haver uma influência positiva com redução da progressão da doença, já que possibilita detectar lesões subcentimétricas. No longo prazo, alguns pacientes poderão inclusive alcançar a cura e, em outros, poderá haver perspectiva de postergar o tratamento sistêmico.

\section{Referências}

1. Siegel R, Ma J, Zou Z et al. Cancer statistics, 2014. CA Cancer J Clin 2014;64:9-29.

2. Boorjian SA, Thompson RH, Tollefson MKetal.Longterm risk of clinical progression after biochemical recurrence following radical prostatectomy: the impact of time from surgery to recurrence. Eur Urol 2011;59:893-9.

3. Mottet N, Bellmunt J, Bolla M et al. EAU guidelines on prostate cancer. Part II: Treatment of advanced, relapsing, and castration-resistant prostate cancer. Eur Urol 2011;59:572-83.

4. Suardi N, Gandaglia G, Gallina A et al. Long-term outcomes of salvage lymph node dissection for clinically recurrent prostate cancer: results of a single-institution series with a minimum follow-up of 5 years. Eur Urol 2015;67:299-309.
5. Abdollah F, BrigantiA, Montorsi F et al. Contemporary role of Salvage Lymphadenectomy in patients with recurrence following radical prostatectomy. Eur Urol 2015;67:839-49.

6. Maurer T, Graefen M, van der Poel H et al. ProstateSpecific membrane antigen-guided surgery. J Nucl Med 2020;6:6-12.

7. Meijer HJ, Fortuin AS, van Lin EN, et al. Geographical distribution of lymph node metastases on MR lymphography in prostate cancer patients. Radiother Oncol. 2013;106:59-63.

8. Knipper S, Tilki D, Mansholt J, Berliner C, Bernreuther $\mathrm{C}$, Steuber T. Metastases-yield and prostate-specific antigen kinetics following salvage lymph node dissection for prostate cancer: A comparison between conventional surgical approach and prostate-specific membrane antigen-radioguided Surgery. Eur Urol Focus 2018.

9. Maurer T, Robu S, Schottelius $M$ et al. $99 \mathrm{~m}$ Technetium-based prostate-specific membrane antigen-radioguided surgery in recurent prostate cancer. Eur Urol 2018.pii:S0302-2838(18)30189-1.

10. Rauscher I, Düwel C, Wirtz M et al. Value of (111) In prostate-specific membrane antigen (PSMA)radioguided surgery for salvage lymphadenectomy in recurrent prostate cancer: correlation with histopathology and clinical follow-up. BJU Int 2017;120(1):40-47.

11. Rigatti P, Suardi N, Briganti A et al. Pelvic/ retroperitoneal salvage lymph node dissection for patients treated with radical prostatectomy with biochemical recurrence and nodal recurrence detected by $[11 \mathrm{C}]$ choline positron emission tomography/ computed tomography. Eur Urol 2011;60:935-43. 